\title{
Squamous cell carcinoma of the uterine cervix associated with osteoclast-like giant cells: A case report and review of the literature
}

\author{
GUOHUA YU ${ }^{1,2^{*}}$, CHUNHUA LIN ${ }^{3 *}$, WEI WANG ${ }^{2}$, YEKUN HAN ${ }^{4}$, GUIMEI QU ${ }^{2}$ and TINGGUO ZHANG ${ }^{1}$ \\ ${ }^{1}$ Department of Pathology, Shandong University School of Medicine, Jinan, Shandong 250012; \\ Departments of ${ }^{2}$ Pathology and ${ }^{3}$ Surgery, Affiliated Yantai Yuhuangding Hospital, Medical College of Qingdao University, \\ Yantai, Shangdong 264000; ${ }^{4}$ Department of Pharmacology, Affiliated Hospital, Binzhou Medical College, \\ Binzhou, Shandong 256603, P.R. China
}

Received February 1, 2014; Accepted July 1, 2014

DOI: $10.3892 / \mathrm{ol} .2014 .2384$

\begin{abstract}
Squamous cell carcinoma is a common malignant tumor of the uterine cervix. The present study reports the case of squamous cell carcinoma of the uterine cervix with osteoclast-like giant cells (OGCs) in an 84-year-old female who had suffered from irregular vaginal bleeding for one month. Colposcopy was performed and a cauliflower-like mass was identified in the front lip of the uterine cervix. Biopsy was then performed, and the tumor was found to be composed of epithelial cell nests, ranging in size. The neoplastic cells exhibited unclear boundaries and eosinophilic cytoplasm. Additionally, the nuclei were atypical and mitosis was observed. Among the epithelial nests, there were numerous OGCs with abundant eosinophilic cytoplasm, as well as multinucleation with bland nuclei. By immunohistochemical staining, the epithelial cells were positive for cytokeratin, while negative for CD68 and vimentin. By contrast, the immunophenotype of the OGCs was the exact opposite. Based on the histological characters, a diagnosis of squamous cell carcinoma of the uterine cervix associated with OGCs was made. Considering the age of the patient, radiotherapy was administered. The patient succumbed to brain metastasis of the tumor after eight months of follow-up.
\end{abstract}

\section{Introduction}

Osteoclast-like giant cells (OGCs) have been described in numerous types of malignant tumors, such as pancreatic,

Correspondence to: Professor Tingguo Zhang, Department of Pathology, Shandong University School of Medicine, 44 Wenhua Xi Road, Jinan, Shandong 250012, P.R. China

E-mail: tingguozh@163.com

${ }^{*}$ Contributed equally

Key words: uterine cervix, squamous cell carcinoma, osteoclast-like giant cells breast, renal, skin, stomach and urinary bladder (1-6). However, OGCs have rarely been found in association with uterine cervix neoplasm. To the best of our knowledge, only three cases of squamous cell carcinoma of the uterine cervix with OGCs have been reported in the English literature $(7,8)$. In the three previous cases, all patients were elderly females ( $\geq 60$ years old) and irregular postmenopausal bleeding was the most common clinical symptom observed. The three patients succumbed to the disease within 14 months of surgery or treatment with radiochemotherapy $(7,8)$. Therefore, the origination and prognostic significance of OGCs in squamous cell carcinoma of uterine cervix are poorly understood. The present study reports a fourth case of squamous cell carcinoma of the uterine cervix associated with OGCs, and the clinicopathological features are discussed. In this study, the case of an 84-year-old female with irregular vaginal bleeding is presented. A cauliflower-like mass was identified in the front lip of the uterine cervix by colposcopy. The patient received palliative radiotherapy and succumbed to brain metastasis after eight months of follow-up. Written informed consent was obtained from the patient for publication of this case report and any accompanying images.

\section{Case report}

Clinical data. An 84-year-old post-menopausal female presented to the Department of Gynaecology at Laishan People's Hospital (Yantai, China) with irregular vaginal bleeding of one month, and was then sent to the Department of Gynaecology at the Affiliated Yantai Yuhuangding Hospital (Yantai, China). Colposcopy was performed and a cauliflower-like mass was identified in the front lip of the uterine cervix. The neoplasm was $\sim 5 \mathrm{~cm}$ in diameter and fragile in texture. Abdominal ultrasound examination showed multiple swollen lymph nodes surrounding the great vessels. The blood counts, clinical biochemistry, urine analysis and endocrine profile were all within normal ranges.

Pathological findings. Biopsy was performed and histological findings showed that epithelial cell nests, ranging in size, had 
A

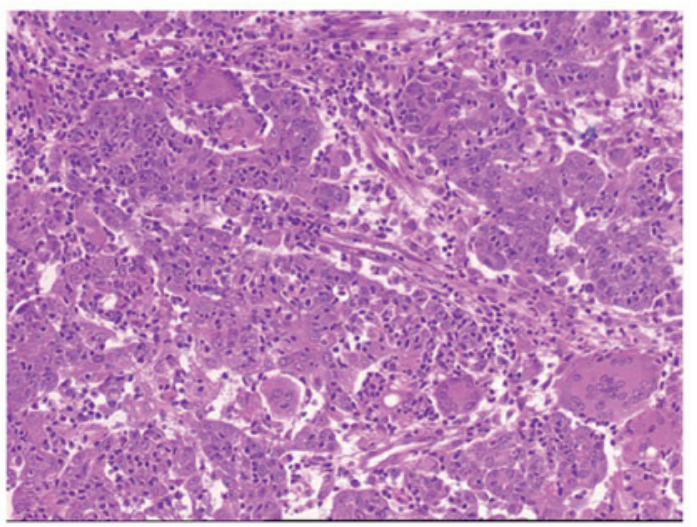

B

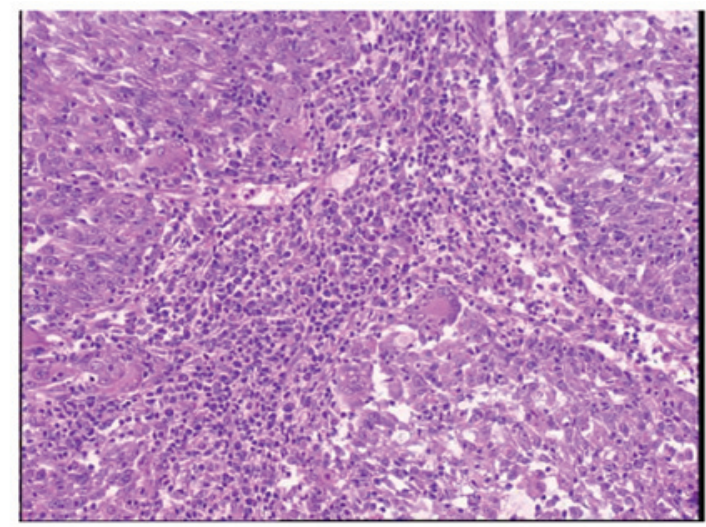

Figure 1. Histological features of the tumor. (A) Irregular epithelial cell nests show infiltrative growth, and there are numerous OGCs among the nests. The nuclei of the epithelial cells are atypical and mitosis is evident, while the nuclei of the OGCs are bland and monomorphic. (B) Numerous lymphatic cells are positive for CD3, mostly among the epithelial cell nests and neoplastic stroma, as shown by immunohistochemical staining. Hematoxylin and eosin staining; magnification, x100. OGCs, osteoclast-like giant cells.

A

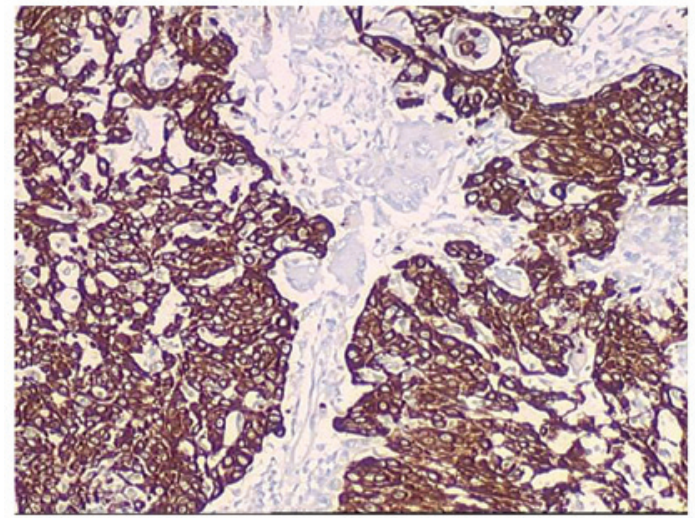

B

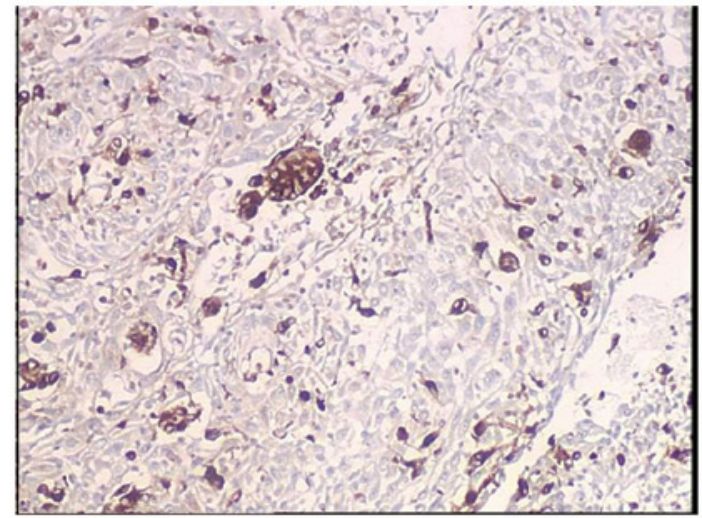

Figure 2. Immunohistochemical features of the OGCs. The OGCs are (A) negative for cytokeratin and (B) positive for CD68 (magnification, x100). OGCs, osteoclast-like giant cells.

infiltrated the stroma. The neoplastic cells presented unclear boundaries and eosinophilic cytoplasm. In addition, the nuclei were atypical and mitosis was observed. There were no obvious keratin pearls or intercellular bridges. Among the epithelial cell nests, there were numerous OGCs with abundant eosinophilic cytoplasm and multinucleation. The nuclei were bland, monomorphic and round or oval in shape. The absence of features of cytological atypia and mitosis distinguished these nuclei from those of the neoplastic cells (Fig. 1A). There were numerous lymphatic cells in the epithelial cell nests and neoplastic stroma (Fig. 1B).

Immunohistochemical findings. Using immunohistochemical techniques, the neoplastic cells were identified to be positive for cytokeratin (Fig. 2A); whereas the OGCs were positive for CD68 (Fig. 2B) and vimentin, but negative for cytokeratin. The majority of lymphatic cells were positive for CD3 and only a small number of lymphocytes expressed CD20. On the basis of the clinical manifestations, histological features and immunohistochemical findings, a diagnosis of non-keratinizing squamous carcinoma of the uterine cervix with OGCs was made. With consideration of the patient's age, the surgical treatment was cancelled with the patient's consent and radiotherapy was administered instead. The total follow-up period was eight months. The patient was alive and the size of neoplasm had decreased prior to the end of radiotherapy, however, the patient succumbed to brain metastasis of the tumor at eight months of follow-up.

\section{Discussion}

A number of studies have shown that OGCs are a unique type of microphage emerging from the mesenchymal tissue, with similar histological features to osteoclasts $(3,5,9,10)$. However, it was previously thought that OGCs were a type of tumor cell that originated from the epithelium (2). In the present case, the OGCs were bland and lacked atypia. Immunohistochemically, the OGCs were positive for CD68 and vimentin, but negative for cytokeratin. These results support the hypothesis that OGCs may simply be immunoreactive cells derived from the mesenchymal tissue. In this case, it is important to distinguish OGCs from multinucleated cells, which are known to indicate a poor prognosis (11). The cells of giant cell tumors exhibit nuclei with hyperchromatism and pleomorphism, while OGCs are an immunoreactive component with uniform chromatin and no obvious atypia (11).

OGCs have been described in numerous types of malignant tumors, such as pancreatic, breast, renal, skin, stomach and 


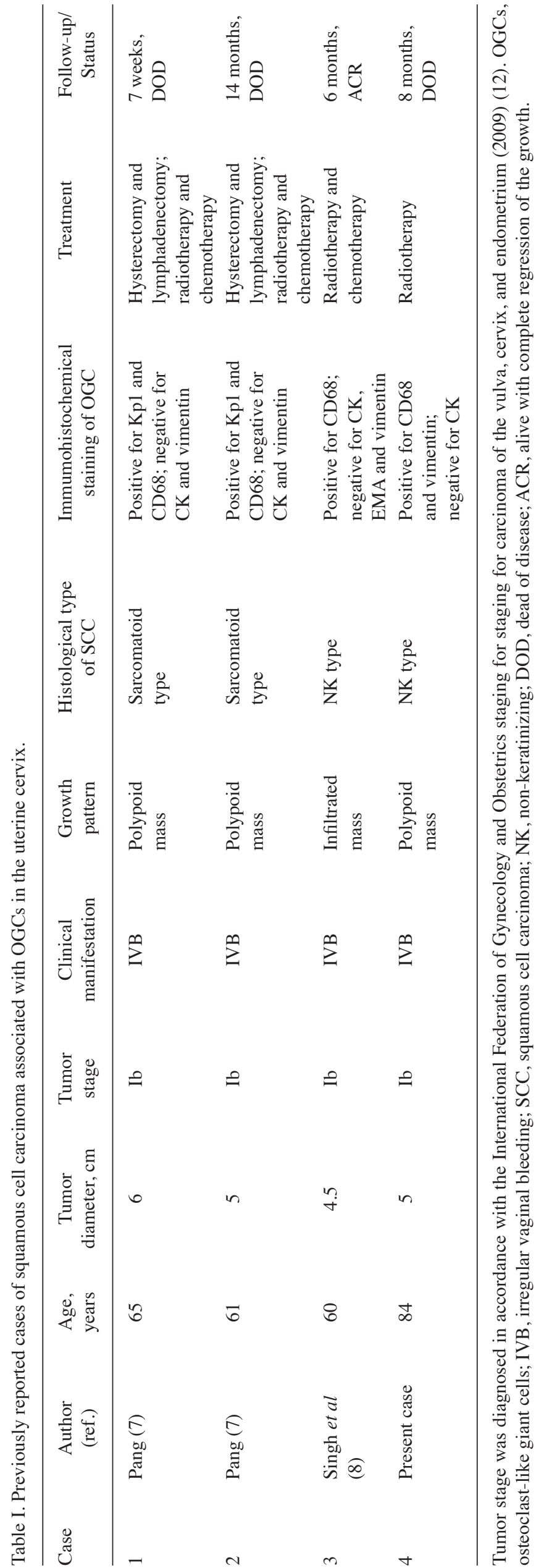

urinary bladder (1-6). The reason for the association of OGCs with these tumor types may be the chemotaxis of tumor cells or inflammatory cells. In the present case, numerous lymphocytes, among which $\mathrm{T}$ lymphocytes were predominant, were identified in the epithelial cell nests and the neoplastic stroma. Therefore, this finding adds support to the abovementioned theory.

To the best of our knowledge, only three cases of squamous cell carcinoma associated with OGCs in the uterine cervix have been reported $(7,8)$, and the clinical information is presented in Table I. All the patients were elderly females $>60$ years of age, and irregular post-menopausal bleeding was the common clinical manifestation. In three cases, the mass showed extruding growth, while one case showed an infiltrated neoplasm. The tumor diameters ranged from 4.5 to $6 \mathrm{~cm}$, and the clinical stage was Ib in all cases according to the International Federation of Gynecology and Obstetrics staging for carcinoma of the vulva, cervix, and endometrium (2009) (12). The type of squamous cell carcinoma which was accompanied by OGCs was sarcomatoid type or non-keratinizing type, which are poorly differentiated types of squamous cell carcinoma. Three patients died within 14 months of surgery or radio-chemotherapy. One patient survived, but was followed up for a shorter time period. Although OGCs may be immunoreactive cells, the presence of OGCs in squamous cell carcinoma of the uterine cervix appears to be an indicator of poor prognosis. However, the type of primary tumor appears to be the main determinant of prognosis, and additional clinical data are required to determine the significance of these findings.

\section{Acknowledgements}

This study was supported by the Project of Administration of Traditional Chinese Medicine of Shandong Province (grant no. 2009222).

\section{References}

1. Molberg KH, Heffess C, Delgado R and Albores-Saavedra: Undifferentiated carcinoma with osteoclast-like giant cells of the pancreas and periampullary region. Cancer 82: 1279-1287, 1998.

2. Borg-Grech A, Morris JA, Eyden BP: Malignant osteoclastoma-like giant cell tumor of the renal pelvis. Histopathology 11: 415-425, 1987.

3. Shishido-Hara Y, Kurata A, Fujiwara M, et al: Two cases of breast carcinoma with osteoclastic giant cells: Are the osteoclastic giant cells pro-tumoural differentiation of macrophages? Diagn Pathol 5: 55, 2010.

4. Willems S, Carneiro F, Geboes K: Gastric carcinoma with osteoclast-like giant cells and lymphoepithelioma-like carcinoma of the stomach: two of a kind? Histopathology 47: 331-333, 2005.

5. Wooff J, Werner D, Murphy J and Walsh N: Osteoclast-like giant cell reaction associated with cutaneous squamous cell carcinoma: a report of 2 cases and review of the literature. Am J Dermatopathol 31: 282-287, 2009.

6. Kemal Behzatoğlu, Haydar Durak, Şule Canberk, et al: Giant cell tumor-like lesion of the urinary bladder: a report of two cases and literature review; giant cell tumor or undifferentiated carcinoma? Diagn Patho 4: 48, 2009.

7. Pang LC: Sarcomatoid squamous cell carcinoma of the uterine cervix with osteoclast-like giant cells: report of two cases. Int J Gynecol Pathol 17: 174-177, 1998.

8. Singh M, Singh S, Mahajan N and Khurana N: Osteoclastic giant cell rich carcinoma cervix: a rare entity. J Obstet Gynaecol 32: 499-501, 2012.

9. Li XM, Lin XY, Xu HT, et al: Mediastinal epithelioid hemangioendothelioma with abundant spindle cells and osteoclast-like giant cells mimicking malignant fibrous histiocytoma. Diagn Pathol 8: 103, 2013. 
10. Suh S, Jung CK, Lee YS, et al: Xp11.2 translocation renal cell carcinoma associated with non-neoplastic osteoclast-like giant cells. Pathol Int 63: 336-338, 2013.

11. Singhal A, Shrago SS, Li SF, et al: Giant cell tumor of the pancreas: a pathological diagnosis with poor prognosis. Hepatobiliary Pancreat Dis Int 9: 433-437, 2010.

12. Pecorelli S: Revised FIGO staging for carcinoma of the vulva, cervix, and endometrium. Int J Gynaecol Obstet 105: 103-104, 2009. 